\title{
Turismo científico una alternativa para conservar el Patrimonio Del CIPCA, Amazonia - Ecuador
}

\author{
Scientific tourism an alternative to conserve CIPCA heritage, Amazonia - Ecuador \\ Paola Silva ${ }^{1}$, Germania Gamboa $^{1}$, Dunia Chávez \\ 1 Universidad Estatal Amazónica, Pastaza, Ecuador. \\ Correspondencia: paolisilva28@gmail.com,mgamboa@uea.edu.ec,dchavez@uea.edu.ec \\ Teléfono: 0987975327
}

Rec.: 14.05.2019. Acept.: 18.10.2019.

Publicado el 31 de diciembre de 2019

\section{Resumen}

$\mathrm{L}$ a investigación se enmarcó en la identificación del patrimonio natural y cultural del Centro de Investigación Posgrado y Conservación Amazónica (CIPCA), en la selección de atractivos con potencial científico y en el análisis de los criterios que inciden en su potencial. El propósito radica en enlazar al turismo con la ciencia, en una modalidad que fomenta una cultura científica, ideal para territorios frágiles y que cuidan del medio ambiente. De esta manera insertar al CIPCA en la actividad turística enfocada, en el descubrimiento de su patrimonio, conformado por unidades socios ambientales anexados a proyectos de investigación. La investigación se desarrolló en CIPCAUEA. La identificación del patrimonio se realizó en base a la metodología: MINTUR (2017a), que establece una jerarquización de los atractivos turísticos en base a las condiciones que presentan. La selección de los atractivos con potencial científico se desarrolló a través del criterio de expertos y método Delphi. El análisis de la incidencia de los criterios con el potencial científico de los atractivos se realizó con estadística descriptiva, tablas de contingencia y la prueba Chi cuadrado. Los resultados obtenidos fueron: 14 manifestaciones culturales y 2 naturales, en total 16 atractivos turísticos; de los cuales 2 atractivos responden a jerarquía II y 14 a jerarquía I. Mediante la valoración se estableció que el CIPCA cuenta con 9 atractivos culturales con mediano nivel de potencial científico, que expone la necesidad en el fortalecimiento de los criterios con alta incidencia en la determinación del potencial científico de los atractivos, los cuales son: valor pedagógico, producción científica, recurso humano, infraestructura y equipamiento tecnológico.

Palabras clave: Turismo alternativo, ciencia y ocio, centros de investigación, turismo educativo, turismo investigativo.

\begin{abstract}
$\mathbf{T}$ the research focused on the identificaction of natural and cultural heritage with scientific potential in Centro de Investigación Posgrado y Conservación Amazónica (CIPCA), in the selection of places with scientific potential and in the analysis of the criteria that affect their potential. The purpose is to link tourism with science, in a way that fosters a scientific culture, ideal for fragile and environmental friendly territories. In this way insert CIPCA into the tourist activity focused on the discovery of its heritage. This is consisting of socio-environmental units attached to research projects. The research was developed at CIPCA-UEA. The identification of heritage was carried out based on the methodology: MINTUR (2017a), which establishes a hierarchy of tourist attractions based on the conditions they present. The selections of the attractions with scientific potential, were developed through the criteria of experts and Delphi method. The analysis of the incidence of the criteria with the attractions' scientific potential was performed with Descriptive Statistics, Contingency tables and Chi-square test. The results were: 14 cultural and 2 natural attractions, 16 tourist attractions, of which 2 attractions respond to hierarchy II and 14 to hierarchy I. Through the assessment it was established that CIPCA has 9 cultural attractions with a medium level of scientific potential. These results expose the need to strengthen the criteria with a high incidence in determining the scientific potential of the attractions, which are: pedagogical value, scientific production, human resources, infrastructure and technological equipment.
\end{abstract}

Keywords: Alternative tourism, science and leisure, research centers, educational tourism, research tourism. 


\section{Introducción}

$\mathrm{E}$ 1 turismo en Ecuador ocupa la tercera posición dentro de las actividades económicas de exportación no petrolera, el $51 \%$ de llegadas corresponden a personas económicamente activas, de las cuales el $25 \%$ son profesionales, científicos e intelectuales (MINTUR, 2017a). La biodiversidad que posee el Ecuador ha sido motivo para que las universidades ofrezcan programas de investigación que conjuntamente con las estaciones biológicas y científicas han generado más de 175 publicaciones en revistas indexadas (Revelo, 2017). De esta manera el patrimonio se convierte en un insumo para los sectores económicos de un país, uno de éstos el turismo (Sandoval, 2017).

El tuirsmo científico para Bourlon et al., (2012) y García y Martínez (2017) es un complemento de varias modalidades tradicionales de turismo, agregándole una dimensión investigativa y de aprendizaje. La clave de esta sincronización turismo y ciencia, es contribuir al desarrollo local de las poblaciones (Salas y Font, 2016). Donde las universidades tienen como misión la producción y difusión del conocimiento científico, a través de la generación de revistas científicas que contribuyan a la transformación y desarrollo de la sociedad (Elías, 2017).

Un trabajo basado en la conservación y sostenibilidad es el que hace hincapié la misión y visión de la Universidad Estatal Amazónica (UEA) y de su Centro de Investigación, Posgrado y Conservación Amazónica (CIPCA), éste último es un escenario conformado por unidades socio ambientales muy diversas que presentan potencialidades en el ámbito turístico (Martín et al., 2014). Gracias a la existencia de proyectos de investigación, el CIPCA se constituye en una plataforma para desarrollar el turismo científico (Ordoñez et al., 2017).

El diagnóstico situacional del CIPCA presenta actividades, programas y proyectos con carencia de visión turística, débil estructura de un producto turístico, la comunidad local se encuentra débilmente empoderada y se desconoce de los beneficios del turismo. Sin embargo, cuenta con varios atractivos turísticos, servicios y facilidades, aptas para desarrollar varias modalidades de turismo rural, una de éstas es el turismo científico (Montoya y Tapia, 2018).

Silva et al., (2017) determinan que el patrimonio que constituye la oferta potencial de turismo científico es la producción científica de la UEA y del CIPCA. Un claro ejemplo son los estudios de manejo y rescate de orquídeas llevados a cabo en el Orquideario y en el sendero ecoturístico del CIPCA, donde se registran 77 especies y 107 taxones diferentes de orquídeas, que son un escenario de aprendizaje para estudiantes e investigadores (Gutiérrez, Asanza y Jalca, 2017). Sobre esta base, el presente artículo estudia si el patrimonio natural y cultural del CIPCA posee potencial científico para el desarrollo de un producto de turismo científico, para lo cual se caracterizó el patrimonio natural y cultural, mediante la identificación de atractivos, selección de atractivos con potencial científico y análisis de los criterios que inciden en el potencial científico de los atractivos.

\section{Materiales y métodos}

$\mathrm{E}^{\mathrm{s}}$ trabajo investigativo se desarrolló en el CIPCA, situado en el cantón Carlos Julio Arosemena Tola, provincia de Napo. La investigación fue de tipo histórica, exploratoria y descriptiva.

La identificación de los atractivos se realizó mediante inventario de atractivos turísticos en base a la metodología del MINTUR (2017b), como técnica se empleó la observación y como instrumento la ficha de inventario; la ponderación de criterios y descripción de jerarquías se detallan en los Cuadros 1 y 2 .

La selección y análisis de los atractivos identificados, en vista de la inexistencia de una matriz específica para la valoración de atractivos con potencial científico, se recurrió a la aplicación del criterio de expertos y su procesamiento a través del método Delphi, que comprendió los siguientes pasos:

1. Se seleccionó mediante la técnica de muestreo no probabilístico bola de nieve, un conjunto de 15 expertos, con alto coeficiente de competencia, conocimiento y/o argumentación sobre turismo científico.

2. Se diseñó un instrumento para determinar los indicadores que permitan identificar atractivos turísticos con potencial científico, con sus respectivos rangos de valoración, se tomó como base 21 indicadores propuestos en investigaciones similares por diferentes autores. Se aplicó una prueba piloto y posteriormente su respectiva validación mediante el cálculo del Coeficiente Alfa de Cronbach, obteniendo 0.751 durante el primer análisis con un total de 21 ítems. Posteriormente se eliminaron aquellos con coeficiente de homogeneidad corregida iguales e inferiores a cero. El cuestionario quedó constituido finalmente por 11 ítems y arrojaron un Coeficiente Alfa de Cronbach final de 0,824. Los resultados de la encuesta fueron analizados mediante estadística descriptiva, estableciendo frecuencias y el cálculo de la media en el programa SPSS versión 23. Los 11 ítems mejor puntuados, están ubicados según su nivel de importancia en forma descendente, con su respectiva ponderación de criterios, como se aprecia en el cuadro 3. 
3. La ponderación de criterios, se realizó en base a la escala de evaluación numérica propuesta por Sandoval (2006), como se describe en el cuadro 4. Esto permitió seleccionar de todos los atractivos turísticos identificados y jerarquizados en base al cuadro 2, en aquellos que alcanzan un nivel de potencial científico.

Para el análisis de los criterios que inciden en el potencial científico de los atractivos, se aplicó la estadística descriptiva, que permitió trabajar con datos cuantitativos. Se aplicaron las tablas de contingencia y las pruebas estadísticas del Chi-cuadrado, cuyo P-Valor debe ser $<0.05$ y el Coeficiente de Contingencia con valor $\geq 0.30$, que en conjunto determinan la fuerte relación entre variables cualitativas, permitiendo aceptar o rechazar la hipótesis de investigación.

Cuadro 1. Ponderación de criterios. Atractivos naturales y culturales

\begin{tabular}{lc}
\hline Criterios de Valoración & Ponderación \\
\hline A. Accesibilidad y conectividad & 18 \\
B. Planta turística/complementarios & 18 \\
C. Estado de conservación e integración sitio/entorno & 14 \\
D. Higiene, seguridad turística & 14 \\
E. Políticas y regulaciones & 10 \\
F. Actividades que se practican en el atractivo & 9 \\
G. Difusión, medios de promoción y comercialización & 7 \\
H. Registro de visitante y afluencia & 5 \\
I. Recursos humanos & 5 \\
\hline TOTAL & $\mathbf{1 0 0}$ \\
\hline
\end{tabular}

Nota. Adaptado de "Metodología para la jerarquización de atractivos y generación de espacios turísticos del Ecuador 2017.1ra Parte" (2da ed.), por Ministerio de Turismo, Quito, octubre 2017.

Cuadro 2. Rangos y descripción de jerarquías y rangos

\begin{tabular}{|c|c|}
\hline Jerarquía y Rangos & Criterios de Valoración \\
\hline $\begin{array}{l}\text { Jerarquía IV } \\
86-100\end{array}$ & $\begin{array}{l}\text { Atractivo excepcional y de alta significación para el mercado turístico internacional, } \\
\text { capaz por sí solo de motivar una importante corriente de visitantes (actual o potencial). }\end{array}$ \\
\hline $\begin{array}{l}\text { Jerarquía III } \\
61-85\end{array}$ & $\begin{array}{l}\text { Atractivo con rasgos excepcionales, capaz de motivar por sí solo o en conjunto con } \\
\text { otros atractivos contiguos, una corriente actual o potencial de visitantes nacionales o } \\
\text { extranjeros. }\end{array}$ \\
\hline $\begin{array}{l}\text { Jerarquía II } \\
36-60\end{array}$ & $\begin{array}{l}\text { Atractivo con algún rasgo llamativo, capaz de interesar a visitantes que hubiesen } \\
\text { llegado a la zona por otras motivaciones turísticas, o de motivar corrientes turísticas } \\
\text { nacionales. }\end{array}$ \\
\hline $\begin{array}{l}\text { Jerarquía I } \\
11-35\end{array}$ & $\begin{array}{l}\text { Atractivo que presenta condiciones mínimas para generar productos, elemento que } \\
\text { complementa a otros de mayor jerarquía. }\end{array}$ \\
\hline $\begin{array}{l}\text { Recurso } \\
0-10\end{array}$ & $\begin{array}{l}\text { Es un elemento natural o cultural que pueden motivar el desplazamiento, pero no se } \\
\text { encuentran todavía incorporados en la dinámica turística, ni cuenta con ningún tipo de } \\
\text { infraestructura de apoyo. }\end{array}$ \\
\hline
\end{tabular}

Nota. Adaptado de "Metodología para la jerarquización de atractivos y generación de espacios turísticos del Ecuador 2017.1ra Parte" (2da ed.), por Ministerio de Turismo, Quito, octubre 2017. 
Cuadro 3. Matriz para valoración del potencial de turismo científico

\begin{tabular}{|c|c|c|c|}
\hline \multirow[b]{2}{*}{ Criterios } & \multicolumn{3}{|c|}{ Valor numérico } \\
\hline & $\begin{array}{c}\text { Mayor potencialidad } \\
3\end{array}$ & 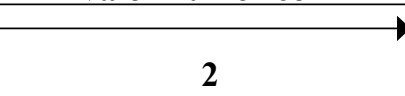 & $\begin{array}{l}\text { Menor potencialidad } \\
1\end{array}$ \\
\hline $\begin{array}{l}\text { 1.Actitud de actores } \\
\text { locales }\end{array}$ & $\begin{array}{l}\text { Total aceptación de } \\
\text { las nuevas actividades } \\
\text { propuestas con mirada } \\
\text { científica por parte de la } \\
\text { comunidad }\end{array}$ & $\begin{array}{l}\text { Mediana aceptación de } \\
\text { las nuevas actividades } \\
\text { propuestas con mirada } \\
\text { científica por parte de la } \\
\text { comunidad }\end{array}$ & $\begin{array}{l}\text { Reducida aceptación de } \\
\text { las nuevas actividades } \\
\text { propuestas con mirada } \\
\text { científica por parte de la } \\
\text { comunidad }\end{array}$ \\
\hline 2. Valor interpretativo & $\begin{array}{l}\text { Alta factibilidad que } \\
\text { tienen los recursos para } \\
\text { fortalecer la sensibilización } \\
\text { sobre la importancia } \\
\text { de la conservación } \\
\text { de los ecosistemas y } \\
\text { para la transmisión de } \\
\text { conocimientos }\end{array}$ & $\begin{array}{l}\text { Mediana factibilidad } \\
\text { que tienen los recursos } \\
\text { para fortalecer la } \\
\text { sensibilización sobre } \\
\text { la importancia de la } \\
\text { conservación de los } \\
\text { ecosistemas y para } \\
\text { la transmisión de } \\
\text { conocimientos }\end{array}$ & $\begin{array}{l}\text { Poca factibilidad que } \\
\text { tienen los recursos } \\
\text { para fortalecer la } \\
\text { sensibilización sobre } \\
\text { la importancia de la } \\
\text { conservación de los } \\
\text { ecosistemas y para } \\
\text { la transmisión de } \\
\text { conocimientos }\end{array}$ \\
\hline $\begin{array}{l}\text { 3. Viabilidad } \\
\text { económica }\end{array}$ & $\begin{array}{l}\text { Viable relación costo- } \\
\text { beneficio para su desarrollo, } \\
\text { gestión y mantenimiento }\end{array}$ & $\begin{array}{l}\text { Posible relación costo- } \\
\text { beneficio para su } \\
\text { desarrollo, gestión y } \\
\text { mantenimiento }\end{array}$ & $\begin{array}{l}\text { Reducida relación } \\
\text { costo-beneficio para su } \\
\text { desarrollo, gestión y } \\
\text { mantenimiento }\end{array}$ \\
\hline 4. Recurso humano & $\begin{array}{l}\text { Existencia de } \\
\text { investigadores con títulos } \\
\text { de cuarto nivel y más de } \\
\text { diez años de experiencia }\end{array}$ & $\begin{array}{l}\text { Existencia de } \\
\text { investigadores con títulos } \\
\text { de cuarto nivel y más de } \\
\text { cinco años de experiencia }\end{array}$ & $\begin{array}{l}\text { Existencia de } \\
\text { investigadores de tercer y } \\
\text { cuarto nivel y con más de } \\
\text { dos años de experiencia }\end{array}$ \\
\hline 5. Atracción & $\begin{array}{l}\text { Alto valor estético, emotivo } \\
\text { y perceptual. }\end{array}$ & $\begin{array}{l}\text { Mediano valor estético, } \\
\text { emotivo y perceptual. }\end{array}$ & $\begin{array}{l}\text { Bajo valor estético, } \\
\text { emotivo y perceptual. }\end{array}$ \\
\hline $\begin{array}{l}\text { 6. Redes académicas/ } \\
\text { científicas }\end{array}$ & $\begin{array}{l}\text { De cooperación } \\
\text { internacional }\end{array}$ & De cooperación nacional & De cooperación regional \\
\hline $\begin{array}{l}\text { 7. Divulgación } \\
\text { científica }\end{array}$ & $\begin{array}{l}\text { Libros, capítulos de libros, } \\
\text { producción científica, } \\
\text { ponencias de impacto } \\
\text { mundial }\end{array}$ & $\begin{array}{l}\text { Libros, capítulos de } \\
\text { libros, producción } \\
\text { científica, ponencias de } \\
\text { impacto regional }\end{array}$ & $\begin{array}{l}\text { Libros, capítulos de } \\
\text { libros, producción } \\
\text { científica, ponencias de } \\
\text { impacto local }\end{array}$ \\
\hline $\begin{array}{l}\text { 8. Infraestructura y } \\
\text { equipamiento científico }\end{array}$ & $\begin{array}{l}\text { Espacios en óptimas } \\
\text { condiciones físicas y } \\
\text { tecnológicas }\end{array}$ & $\begin{array}{l}\text { Espacios en buenas } \\
\text { condiciones físicas y } \\
\text { tecnológicas }\end{array}$ & $\begin{array}{l}\text { Espacios en regulares } \\
\text { condiciones físicas y } \\
\text { tecnológicas }\end{array}$ \\
\hline 9. Vinculación & $\begin{array}{l}\text { Existencia de vinculación } \\
\text { con redes, instituciones y/o } \\
\text { asociaciones }\end{array}$ & $\begin{array}{l}\text { Posibilidad de } \\
\text { vinculación en redes, } \\
\text { instituciones y/o } \\
\text { asociaciones }\end{array}$ & $\begin{array}{l}\text { Difícil vinculación en } \\
\text { redes, instituciones, } \\
\text { asociaciones }\end{array}$ \\
\hline 10. Representatividad & $\begin{array}{l}\text { Promueve íntegramente las } \\
\text { prácticas ancestrales locales } \\
\text { su legado e importancia. }\end{array}$ & $\begin{array}{l}\text { Promueve } \\
\text { moderadamente las } \\
\text { prácticas ancestrales } \\
\text { locales su legado e } \\
\text { importancia. } \\
\end{array}$ & $\begin{array}{l}\text { Promueve ligeramente } \\
\text { las prácticas ancestrales } \\
\text { locales su legado e } \\
\text { importancia. }\end{array}$ \\
\hline 11. Valor pedagógico & $\begin{array}{l}\text { Ilustra contenidos } \\
\text { curriculares para un público } \\
\text { especializado o de nivel } \\
\text { superior }\end{array}$ & $\begin{array}{l}\text { Ilustra contenidos } \\
\text { curriculares para } \\
\text { un público de nivel } \\
\text { educativo medio }\end{array}$ & $\begin{array}{l}\text { Ilustra contenidos } \\
\text { curriculares para público } \\
\text { de cualquier nivel }\end{array}$ \\
\hline
\end{tabular}

Nota. Adaptado de "Ecoturismo: Operación técnica y gestión ambiental”, de Sandoval, E., 2006, p. 89, México: Trillas Recuperado de https:/es.scribd.com/document/55047914/Ecoturismo. "Seleção de geossítios para uso turístico no parque estadual do Ibitipoca/MG (PEI): uma proposta a partir de metodologías de avaliação numérica, Moreira, L., y Rodrigues, S., 2013, p. 37, doi:10.14350/rig.42254. "Proposta Metodológica para a Inventariação do Património Geológico Brasileiro (Tese de Mestrado em Património Geológico e Geoconservação)", de Lima, F., 2008, pp. 70,71. Recuperado de http:/ / www.dct.uminho.pt/mest/pgg/docs/tese_lima.pdf. "Criterios e indicadores para evaluar el potencial del Turismo Científico", de Szmulewicz, P., Veloso, K., y Bourlon, F., 2017, Universidad Austral de Chile y Centro de Investigación en Ecosistemas de la Patagonia, Coyhaique, Chile. 
Cuadro 4. Escala de evaluación para espacios con potencial de turismo científico

\begin{tabular}{cl}
\hline Escala de evaluación & Nivel de Potencial \\
\hline 36 a 30 & Alto potencial \\
29 a 20 & Mediano potencial \\
19 a 10 & Bajo potencial \\
\hline
\end{tabular}

Nota. Fuente Adaptado de "Ecoturismo: Operación técnica y gestión ambiental", de Sandoval, E., 2006, p. 89, México: Trillas.

\section{Resultados y discusión}

$\mathrm{L}$ a identificación del patrimonio natural y cultural del CIPCA, se realizó a través del inventario de atractivos. Se identificaron 16 atractivos turísticos, siendo 14 manifestaciones culturales, esto se debe a la existencia de espacios, donde la intervención humana utilizó los recursos del entorno, para formar programas académicos y productivos especializados en diversos campos. Fueron 2 los atractivos con categoría natural, como se detalla en el cuadro 5. Los atractivos naturales identificados que son dos ríos, se consideran complementarios, según la categoría alcanzada, sin embargo, se revela la existencia de otros recursos que 'pueden sumarse a estos atractivos como lo señala Reyes et al., (2017) al identificar especies de flora y fauna existentes en CIPCA que son de importancia turística.

El $87 \%$ de los atractivos turísticos identificados en el CIPCA obtuvieron jerarquía I, por cuanto presentan condiciones mínimas para generar productos y constituyen elementos que complementan a otros de mayor jerarquía. El 13\% alcanzaron jerarquía II, es decir que son atractivos con rasgos llamativos que son de interés de los visitantes, por lo que actualmente generan visitas por sí solos (MINTUR, 2017b).

En la selección y análisis de espacios identificados con potencial turístico, los 16 atractivos, entre naturales y manifestaciones culturales, fueron valorados en base a cada criterio descrito en la matriz de la tabla 3. En los cuales se asignó un valor de 1 menor potencial, 2 mediano potencial y 3 alto potencial, el valor 0 corresponde a la carencia de las características especificadas en cada criterio. Los puntajes, la sumatoria y los niveles alcanzados por cada atractivo, se detallan en el cuadro 6.Luego de la valoración, en el sumatorio total, se observan siete atractivos con bajo potencial $43.75 \%$, son los programas con débil participación de actores en temas académicos y al pausado trabajo investigativo, así como la escasa inversión en equipamiento e infraestructura. Nueve atractivos con mediano potencial $56.25 \%$, estos pertenecen a la categoría manifestación cultural y constituyen los programas productivos. Al valorar a los 16 atractivos en base a los 11 criterios se observó, en la sumatorio total, que ninguno alcanza una jerarquía alta, sin embargo, se observan puntuaciones altas en ciertos criterios, que inciden favorablemente en determinar que los atractivos poseen mediano potencial científico, además muestra aquellos criterios que requieren de un fortalecimiento.

Para determinar el nivel de incidencia de los 11

\section{Cuadro 5. Jerarquización de atractivos turísticos del CIPCA}

\begin{tabular}{clc}
\hline \multicolumn{1}{c}{ Categoría } & \multicolumn{1}{c}{ Nombre del atractivo } & Jerarquía \\
\hline Manifestaciones culturales & Programa Recursos Acuáticos & I \\
Manifestaciones culturales & Programa Porcino & I \\
Manifestaciones culturales & Programa Pastos y Forrajes & I \\
Manifestaciones culturales & Programa Agroforestal & I \\
Manifestaciones culturales & Programa Avícola & I \\
Manifestaciones culturales & Programa Especies menores & I \\
Manifestaciones culturales & Programa Bovino & I \\
Manifestaciones culturales & Programa Abonos orgánicos & I \\
Manifestaciones culturales & Programa Pequeños Rumiantes & I \\
Manifestaciones culturales & Zoológico Kausarinimi & I \\
Manifestaciones culturales & Herbario ECUAMZ & II \\
Manifestaciones culturales & Programa Plantas medicinales & I \\
Manifestaciones culturales & Jardín Botánico & I \\
Manifestaciones culturales & Estación Alto Piatúa & I \\
Atractivos Naturales & Río Anzu & I \\
Atractivos Naturales & Río Piatúa & II \\
\hline
\end{tabular}


Silva et al., 2019

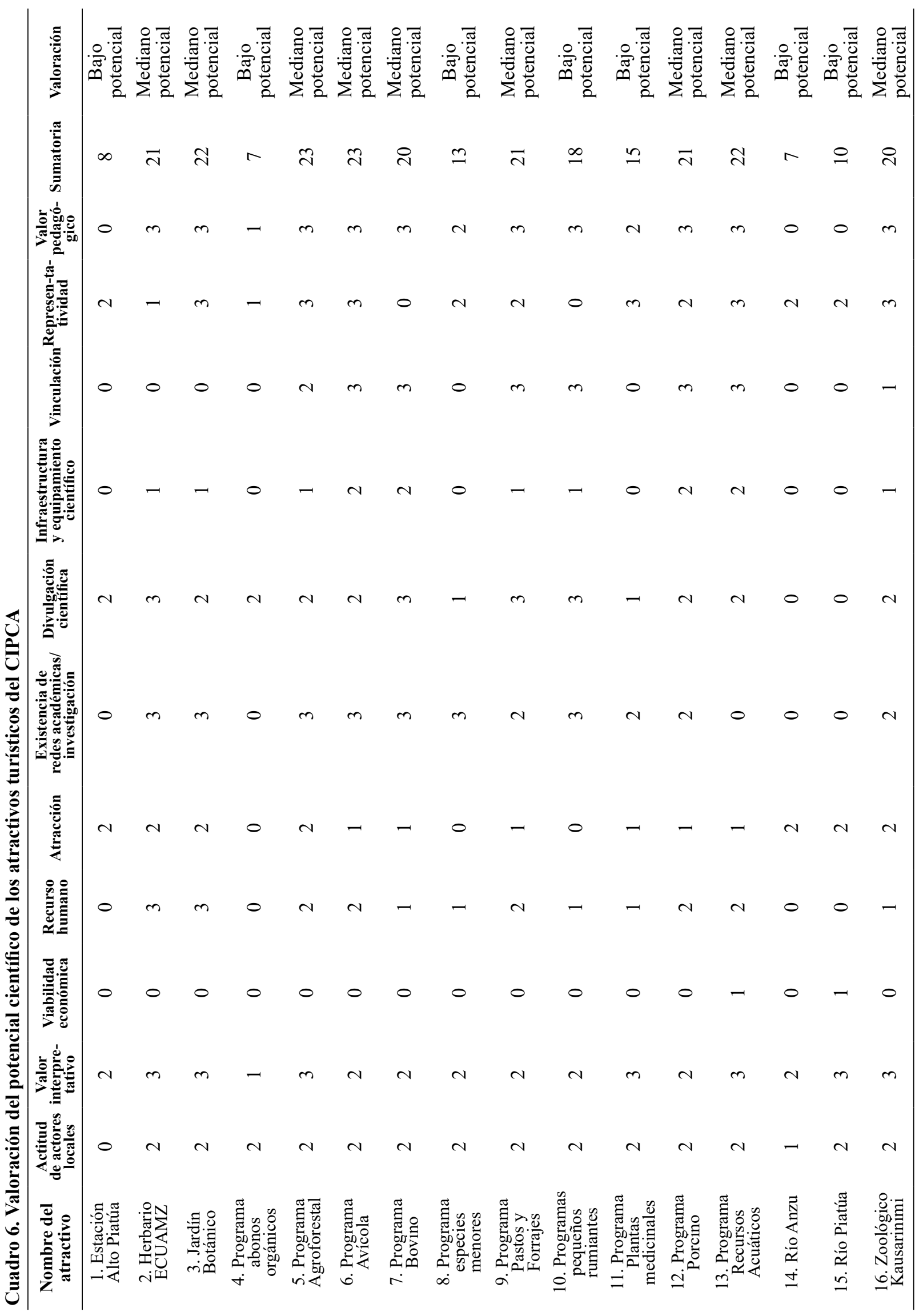


criterios en la existencia o no de potencial científico de los atractivos del CIPCA, se procedió a cruzar las variables: criterios y los puntajes obtenidos por los 16 atractivos en cada uno de ellos. El cruce de variables se realizó a través de las tablas de contingencia y se comprobó con la prueba Chi-cuadrado la alta dependencia entre variables. Luego del análisis estadístico se determinó que fueron 4 los criterios que muestran una estrecha relación, como se describe en los cuadros $7,8,9$ y 10 .

\section{Criterio valor pedagógico}

En el cuadro 7 se observa el 56.3\% de atractivos que alcanzaron niveles altos en el criterio valor pedagógico, esto se debe a la existencia de programas productivos y de conservación con contenidos pedagógicos, creados para brindar apoyo a la docencia y ser espacios donde los estudiantes pongan en práctica sus conocimientos, además cuentan con información para un público especializado o de nivel superior. Ordóñez et al., (2017) hace alusión a que la existencia de los programas didácticos-productivos constituyen un escenario alentador para el desarrollo del turismo científico en CIPCA.

Por otro lado, el $18.8 \%$ que no posee valor pedagógico, representa a los atractivos naturales, los cuáles carecen de planes o programas académicos. El resultado de la prueba no paramétrica Chi-Cuadrado de Pearson, dio un valor de 0.006; del Coeficiente de contingencia se obtuvo un resultado de 0.660 ; valores que confirma la fuerte relación entre las variables valor pedagógico y el nivel en el potencial científico de los atractivos.

\section{Criterio divulgación científica}

Del cruce de variables de la tabla 8 se observa que, de los 9 atractivos identificados con potencial científico, 5 de ellos se ubican en nivel medio $31.3 \%$, debido a que la producción científica (publicaciones de libros, capítulos de libros, artículos científicos, etc.) en su mayor parte son de impacto regional. Los 3 atractivos que no poseen potencial científico en función de la variable divulgación científica, representan a los atractivos naturales, de los cuales no existe producción científica hasta el momento de la investigación.

De la prueba no paramétrica Chi-Cuadrado de Pearson resultó un valor de 0.019; y el Coeficiente de contingencia reflejó un valor de 0.618; valores que confirma que las variables divulgación científica y potencial científico, son dependientes.

\section{Criterio recurso humano}

En el cuadro 9 se observa como resultado del cruce de las variables, que 5 de los 9 atractivos alcanzaron mediano potencial $31.3 \%$. Esto se debe a que los atractivos cuentan con investigadores de cuarto nivel y más de cinco años de experiencia. Por otro lado, 4 atractivos no alcanzaron el potencial científico $25 \%$, tres de ellos pertenecen a la categoría naturales, donde la intervención del recurso humano es limitada y una manifestación cultural que carece de personal especializado.

El cruce de las variables recurso humano y potencial científico, en la prueba Chi cuadrado dio como significancia asintótica el número 0.011 ; y en la prueba no paramétrica Coeficiente de contingencia 0.64. Resultados que confirma la dependencia entre las variables es decir que la presencia de docentes investigadores es un factor fundamental para generar ciencia a partir de los recursos del medio.

Cuadro 7. Tabla de contingencia del criterio valor pedagógico y su incidencia en el potencial científico de los atractivos del CIPCA

\begin{tabular}{|c|c|c|c|c|c|}
\hline & & & \multicolumn{2}{|c|}{$\begin{array}{c}\text { Potencial científico de } \\
\text { atractivos }\end{array}$} & \multirow[t]{2}{*}{ Total } \\
\hline & & & si & no & \\
\hline \multirow{4}{*}{$\begin{array}{l}\text { Divulgación } \\
\text { científica }\end{array}$} & Ninguno & $\%$ del total & $0.0 \%$ & $18.8 \%$ & $18.8 \%$ \\
\hline & Menor potencial & $\%$ del total & $6.3 \%$ & $18.8 \%$ & $25.0 \%$ \\
\hline & $\begin{array}{l}\text { Mediano } \\
\text { potencial }\end{array}$ & $\%$ del total & $31.3 \%$ & $0.0 \%$ & $31.3 \%$ \\
\hline & Mayor potencial & $\%$ del total & $18.8 \%$ & $6.3 \%$ & $25.0 \%$ \\
\hline \multicolumn{2}{|r|}{ Total } & $\%$ del total & $56.3 \%$ & $43.8 \%$ & $100.0 \%$ \\
\hline
\end{tabular}


Cuadro 8. Tabla de contingencia del criterio divulgación científica y potencial científico de los atractivos del CIPCA

\begin{tabular}{|c|c|c|c|c|c|}
\hline & & & \multicolumn{2}{|c|}{$\begin{array}{l}\text { Potencial científico de } \\
\text { atractivos }\end{array}$} & \multirow[t]{2}{*}{ Total } \\
\hline & & & si & no & \\
\hline \multirow{4}{*}{ Valor pedagógico } & Ninguno & $\begin{array}{l}\% \text { del } \\
\text { total }\end{array}$ & $0.0 \%$ & $18.8 \%$ & $18.8 \%$ \\
\hline & Menor potencial & $\begin{array}{l}\% \text { del } \\
\text { total }\end{array}$ & $0.0 \%$ & $6.3 \%$ & $6.3 \%$ \\
\hline & $\begin{array}{l}\text { Mediano } \\
\text { potencial }\end{array}$ & $\begin{array}{l}\% \text { del } \\
\text { total }\end{array}$ & $0.0 \%$ & $12.5 \%$ & $12.5 \%$ \\
\hline & Mayor potencial & $\begin{array}{l}\% \text { del } \\
\text { total }\end{array}$ & $56.3 \%$ & $6.3 \%$ & $62.5 \%$ \\
\hline Total & & $\begin{array}{l}\% \text { del } \\
\text { total }\end{array}$ & $56.3 \%$ & $43.8 \%$ & $100.0 \%$ \\
\hline
\end{tabular}

Cuadro 9. Tabla de contingencia del criterio recurso humano y potencial científico de los atractivos del CIPCA

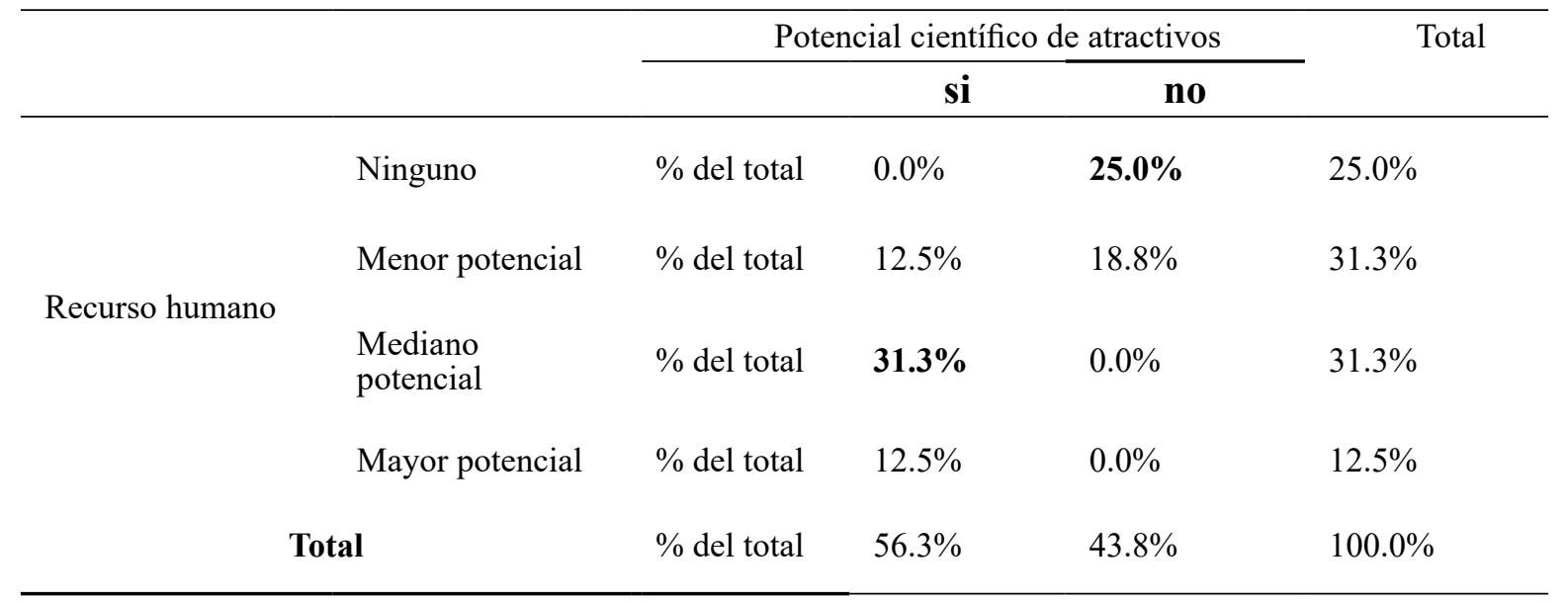

Cuadro 10. Tabla de contingencia de la variable infraestructura y equipamiento científico y potencial científico de los atractivos del CIPCA

\begin{tabular}{|c|c|c|c|c|c|}
\hline & & & \multicolumn{2}{|c|}{$\begin{array}{l}\text { Potencial científico de } \\
\text { atractivos }\end{array}$} & \multirow[t]{2}{*}{ Total } \\
\hline & & & si & no & \\
\hline \multirow{3}{*}{$\begin{array}{l}\text { Infraestructura y } \\
\text { equipamiento científico }\end{array}$} & Ninguno & $\begin{array}{l}\% \text { del } \\
\text { total }\end{array}$ & $0.0 \%$ & $37.5 \%$ & $37.5 \%$ \\
\hline & Menor potencial & $\begin{array}{l}\% \text { del } \\
\text { total }\end{array}$ & $31.3 \%$ & $6.3 \%$ & $37.5 \%$ \\
\hline & Mediano potencial & $\begin{array}{l}\% \text { del } \\
\text { total }\end{array}$ & $25.0 \%$ & $0.0 \%$ & $25.0 \%$ \\
\hline Total & & $\begin{array}{c}\% \text { del } \\
\text { total }\end{array}$ & $56.3 \%$ & $43.8 \%$ & $100.0 \%$ \\
\hline
\end{tabular}




\section{Criterio infraestructura y equipamiento científico}

En el cuadro 10, se observa como resultados del cruce de las variables, que 5 atractivos turísticos del CIPCA $31.3 \%$, poseen menor potencial, 4 atractivos $25 \%$, con mediano potencial, esto responde a que las instalaciones y equipamiento tecnológico con los que cuentan los diferentes atractivos, se encuentran en regulares condiciones y 6 atractivos $37.5 \%$, sin potencial científico en este criterio.

Sin embargo, del cálculo de la prueba no paramétrica Chi-Cuadrado de Pearson, se obtuvo un valor de 0.002 y de la prueba no paramétrica Coeficiente de contingencia, valor igual a 0.664 . Números que confirman la relación de dependencia entre las variables infraestructura y equipamiento científico con el nivel de potencial científico de un atractivo.

La infraestructura al hablar de turismo debe tener en cuenta procesos logísticos adecuados y sostenibles para los recursos naturales y culturales, enlazados al compromiso social, económico y ambiental (Ascuntar et al., 2017). Mullo et al., (2017) señala que el turismo indistintamente del enfoque que posea, es una actividad productiva e interterritorial, siendo la infraestructura clave en el uso y comercialización de sus servicios.

\section{Conclusiones}

$\mathrm{C}$ IIPCA cuenta con 16 atractivos turísticos, de los cuales 14 son manifestaciones culturales y 2 atractivos naturales. De éstos, el $87 \%$ de los atractivos turísticos identificados obtuvieron jerarquía I y el 13\% alcanzaron jerarquía II.

En la valoración del potencial científico, de los 16 atractivos identificados, 9 alcanzaron un mediano potencial y siete un bajo potencial. El CIPCA no cuenta con atractivos con alto potencial científico.

En el análisis de los criterios que inciden en el potencial científico de los atractivos, se determinó que la variable valor pedagógico mostró una fuerte relación con el potencial científico de los atractivos, arrojó un P-Valor $=0.006$ y es en la cual los atractivos alcanzaron mayor potencial. Por su parte las variables producción científica y recurso humano, mostraron también fuerte dependencia con un P-Valor $=0.019$ y 0.011 respectivamente, donde los atractivos obtuvieron mediano potencial. En tanto, a pesar de que existe fuerte dependencia entre la variable infraestructura y equipamiento científico con el potencial científico, con $\mathrm{P}$-Valor $=0.002$, los atractivos turísticos obtuvieron menor potencial.

\section{Bibliografía}

Ascuntar, E., Caicedo, J., Guerrero, K., Rosero, M., Vallejo,
Á., y Paz, A. (2017). El Turismo como estrategia de desarrollo económico, social y ambiental. Horizontes empresariales. Boletín de la Facultad de Ciencias Administrativas y Contables. CESMAG, (7), 13-21.

Bourlon , F., Farmer, I., Segura, P., y Osorio, M. (2012). Las ciencias al servicio del Desarrollo Turístico. Creando el Centro para el Turismo Cientifico de la Patagonia. Chile, 1-108.

Elías, J. (2017). La dinámica de las publicaciones universitarias. Procesos editoriales de producción intelectual y de divulgación científica cultural: el caso de Universidad del Magdalena. Jangwa Pana, 16(1), 3137. doi:

García, M., y Martínez, O. (2017). Turismo científico y ciudades del futuro. International Journal of Scientific Management and Tourism, 3(1), 123-130.

Gutiérrez, D., Asanza, M., y Jalca, I. (2017). Manejo y rescate de orquídeas en la Alta Amazonía Ecuatoriana. Hacia un listado de orquídeas epífitas del entorno del CIPCA. III Jornada Iberoamericana en Saludo al Día Mundial del Medio Ambiente-Ecuador 2017, 118-132, Puyo.

Lima, F. (2008). Proposta Metodológica para a Inventariação do Património Geológico Brasileiro. Brasil: (Mestrado em Patrimônio Geológico e Geoconservação) - Escola de Ciências, Universidade do Minho, Minho.

Martín, I., Valencia, G., Velásquez, V., y Navarrete, H. (2014). El CIPCA y su entorno: conociendo sus potencialidades para el desarrollo turístico. Huellas del Sumaco,11, 4246.

Ministerio de Turismo. (2017a). Boletín de Estadisticas Turísticas 2011-2015.

Ministerio de Turismo. (2017b). Metodología para la jerarquización de atractivos y generación de espacios turísticos del Ecuador 2017. Quito: Segunda Edición.

Montoya, J., y Tapia, D. (2018). Zonificación turística del Centro de Investigación, Posgrado y Conservación Amazónica (CIPCA) (Tesis de pregrado). Universidad Estatal Amazónica.

Moreira, L., y Rodrigues, S. (2013). Seleção de geossítios para uso turístico no parque estadual do Ibitipoca/MG (PEI): uma proposta a partir de metodologias de avaliação numérica. Investigaciones Geográficas, Boletín del Instituto de Geografía, (85), 33-46.

Mullo, E., Mendoza, E., y Yumisaca, J. (2017). El impacto de la ciencia y tecnología en la cultura del turismo comunitario: el caso de Salasaca-Ecuador. Revista Ciencias Pedagógicas e Innovación , 5(3), 100-108.

Ordóñez, E., Andrade, C., Auquilla, L., \& Valdés, M. (2017). El Turismo Científico y su contribución al desarrollo local sostenible en Ecuador. Caso de Estudio. Revista DELOS, 10(28).

Revelo, R. (2017). Estudio de los beneficios de tener una Estación Científica en la Amazonía. Revista Publicando, 4(10), 505-519. 
Reyes, M., Vega, A., Ortega, Á., y Manosalvas, C. (2017). Potencial para el Desarrollo del Turismo de Naturaleza en el Centro de Investigación, Posgrado y Conservación de la Biodiversidad Amazónica CIPCA, Universidad Estatal Amazónica (Pastaza-Ecuador). Revista Caribeña de Ciencias Sociales.

Salas, W., y Font, M. (2016). Buenas prácticas en ciencia y tecnología para el desarrollo turístico local. Ecociencia, $3(3), 47-68$.

Sandoval, E. (2006). Ecoturismo: Operación técnica y gestión ambiental. México: Trillas.

Sandoval, L. (2017). Patrimonio cultural y turismo en el Ecuador. Tsafiqui Revista de Investigación Científica, (9), 1-24.

Silva, P., Ruiz E., Valarezo Espinosa C. (2017) La producción científica como patrimonio de la oferta turística en la Amazonia ecuatoriana Análisis temporal CIPCA. Simposio internacional sobre Manejo sostenible de tierras y seguridad alimentaria - Ecuador 2017. Pag 239

Szmulewicz, P., Veloso, K., y Bourlon, F. (2017). Diseño de indicadores para la evaluación del potencial de turismo científico en destinos. Centro de Investigaciones en Ecosistema de la Patagonia y la Universidad Austral de Chile 